\title{
The Effectiveness and Cost-Effectiveness of Web-Based and Home-Based Postnatal Psychoeducational Interventions for First-Time Mothers: Randomized Controlled Trial Protocol
}

Honggu He ${ }^{1 *}$, PhD; Lixia Zhu ${ }^{1 *}$, PhD; Sally Wai Chi Chan ${ }^{2 *}$, PhD; Yap-Seng Chong ${ }^{1}$, PhD; Nana Jiao ${ }^{1}$, MSc; Yiong Huak Chan ${ }^{1 *}, \mathrm{PhD}$; Nan Luo ${ }^{1}, \mathrm{PhD}$; Shefaly Shorey ${ }^{3}, \mathrm{PhD}$

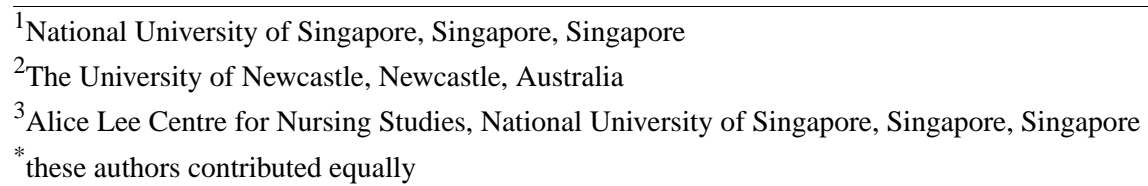

\section{Corresponding Author:}

Shefaly Shorey, $\mathrm{PhD}$

Alice Lee Centre for Nursing Studies

National University of Singapore

Level 2 Clinical Research Centre, Block MD 11, 10 Medical Drive

Singapore, 117597

Singapore

Phone: 6566011294 ext 1294

Fax: 6567767135

Email: nurssh@nus.edu.sg

\section{Abstract}

Background: In addition to recuperating from the physical and emotional demands of childbirth, first-time mothers are met with demands of adapting to their social roles while picking up new skills to take care of their newborn. Mothers may not feel adequately prepared for parenthood if they are situated in an unsupported environment. Postnatal psychoeducational interventions have been shown to be useful and can offer a cost-effective solution for improving maternal outcomes.

Objective: The objective of this study was to examine the effectiveness and cost-effectiveness of Web-based and home-based postnatal psychoeducational programs for first-time mothers on maternal outcomes.

Methods: A randomized controlled three-group pre- and posttests experimental design is proposed. This study plans to recruit 204 first-time mothers on their day of discharge from a public tertiary hospital in Singapore. Eligible first-time mothers will be randomly allocated to either a Web-based psychoeducation group, a home-based psychoeducation group, or a control group receiving standard care. The outcomes include maternal parental self-efficacy, social support, psychological well-being (anxiety and postnatal depression), and cost evaluation. Data will be collected at baseline, 1 month, 3 months, and 6 months post-delivery.

Results: The recruitment ( $\mathrm{n}=204)$ commenced in October 2016 and was completed in February 2017, with 68 mothers in each group. The 6-month follow-up data collection was completed in August 2017.

Conclusions: This study may identify an effective and cost-effective Web-based postnatal psychoeducational program to improve first-time mothers' health outcomes. The provision of a widely-accessed Web-based postnatal psychoeducational program will eventually lead to more positive postnatal experiences for first-time mothers and positively influence their future birth plans.

Trial Registration: International Standard Randomized Controlled Trial Number (ISRCTN): 45202278; http://www.isrctn.com/ISRCTN45202278 (Archived by WebCite at http://www.webcitation.org/6whx0pQ2F).

(JMIR Res Protoc 2018;7(1):e35) doi: 10.2196/resprot.9042

\section{KEYWORDS}

mothers; education; postpartum period; Internet 


\section{Introduction}

The period following a child's birth is considered the most crucial period for mothers as they venture from their current-known reality to an unknown new reality. In addition to recuperating from the physical and emotional demands of childbirth, first-time mothers are concurrently confronted with challenges associated with the demands of adapting to their social roles as new parents [1]. In addition, they are required to pick up new skills to take care of their newborn [2]. The subsequent mastery of these necessary skills at home, without the support from health care professionals, may be challenging for some. This change in role from a nonparent, being only responsible for oneself, to having added responsibility in caring for their newborn could take a toll on first-time mothers if their expectations do not match their experiences [3]. According to the theory of maternal role attainment, a mother's emotional outcome during this phase is very much influenced by the mother's biopsychosocial being, family, and the environment that she is surrounded by [4]. Often, mothers are situated in an unsupported environment and may not feel adequately prepared for motherhood. This presents as a challenge physically, emotionally, and socially, and this is particularly so for first-time mothers [5]. The early postpartum phase presents a critical period where mothers prepare themselves for parenting. A mother's negative psychological status and well-being influence the type of parenting given to their newborn in their developmental stages, consequently impairing the mother-infant relationship and impacting the child's outcome [6].

\section{Postnatal Services in Singapore and Underused Antenatal Classes}

To support mothers, antenatal classes and postnatal support are largely provided by the polyclinics and maternity hospitals in Singapore [7]. Antenatal classes are spread over several weeks and are usually conducted by nurses or midwives from maternity wards or private hospitals. A systematic review revealed that midwifery-led care was associated with better maternal outcomes and should be encouraged [8]. First-time mothers find that being able to communicate with a midwife regarding issues not only helped develop a trusting relationship but also helped to promote choice and control, making them feel more prepared for parenting [9]. Mothers were also satisfied with midwifery-led care [10]. However, antenatal classes usually come with a high price tag in Singapore, which mothers from lower socioeconomic backgrounds may not have access to. In contrast, such classes in certain countries in Western Europe have been offered to mothers without a fee [11]. Research also suggests that the home services provided to mothers are associated with improved emotional maternal outcomes and satisfaction [12].

\section{Challenges Surrounding Postnatal Period}

The average length of hospital stay post delivery is approximately 24 to 72 hours for normal deliveries across all maternity hospitals in Singapore [13]. During the transitional period from hospital to home, mothers face physical challenges, including fatigue from lack of sleep. Mothers often feel a lack of support from 1 to 3 weeks after their discharge [14]. The reality of caring for their newborn only sets in after they are discharged and on their own, and this is when most learning needs are found [15]. Hence, there is a gap in the spectrum of care from the point of discharge back to their homes.

\section{Postnatal Depression}

Postnatal depression (PND) is a depressive illness of mild to moderate severity that occurs during the first postnatal year [16]. It is one of the most common health issues that occurs among postnatal mothers globally, with most mothers experiencing PND in the first 3 months of the postnatal period [17]. In an earlier meta-analysis based on 59 studies, the prevalence of PND was approximately $13 \%$ [18]. In a recent meta-analysis of 18 studies, the overall prevalence was $20 \%$ [19], whereas another meta-analysis of 78 studies reported an overall prevalence of $3.1 \%$ [20]. The prevalence of PND in Singapore is $6.8 \%$ [21]. A local study suggested that mothers might be vulnerable to emotional distress cross-culturally regardless of their cultural postnatal traditions [22].

PND is caused by an interplay of a multitude of psychological factors, including societal expectations, social connectedness, child's temperamental challenges, a mother's coping strategies, and many other life stressors [23]. Physiological causes also include neurochemical imbalances in the brain [24]. If left untreated, it can persist for many years [25], leaving detrimental effects on the mother's recovery period. Moreover, there is an association between the mother's mental health status and maternal-child bonding, which subsequently affects the development of depression in adolescence [6]. The father and the family unit are also inevitably affected, which in turn may affect the mother's social relationships [26,27]. These challenges are important in informing health care providers for developing a service that is essential to the promotion of a positive maternal experience. Such strategies may have an impact on future fertility rates, which is important for Singapore, a country with a rapidly aging population and a very low birth rate.

\section{Social Support, Maternal Self-Efficacy, and Postnatal Depression}

Social support refers to interpersonal relationships that provide individuals with emotional support and self-confidence for maintaining maternal and infant psychological well-being. This form of support facilitates a mother's adjustment into motherhood [28]. It acts as a form of buffer for mothers to mediate stress. Parenting can be psychologically stressful for first-time mothers [29], but good social support from various parties can help ease the transition phase into parenthood. The primary sources of support for a mother come mostly from their partner, other family members, and friends. However, providing support for mothers by nurses and midwives during the early postnatal period is also important, as there have been consistent evidence showing that the availability of social support contributes significantly to a person's ability to self-regulate in the presence of stressors [30-33]. A study conducted in Singapore also underlined the significance of providing competent postnatal care to first-time mothers to improve maternal outcomes [22]. Moreover, barriers to mobilizing support were also present and these include fear of judgment and feeling like a burden [34]. Hence, having a variety of support providers can help protect mothers against the harmful 
psychological effects of stressors related to parenting by enhancing facilitators and reducing barriers to mobilizing support.

Another crucial component in a seamless transition to parenthood for mothers is maternal self-efficacy. Maternal self-efficacy is the belief that a mother holds of her competence in achieving a set of tasks that produces results related to parenting [35]. According to Bandura's theory of self-efficacy, perceptions of self-efficacy come from four main sources of feedback: mastery experience, vicarious experiences, verbal persuasion, and emotional arousal [36]. For example, in the proposed study, mastery experience means the ability to strengthen self-motivation following successes in performing baby care or self-care tasks. Vicarious experiences refers to acquiring a skill through modeling from peer mothers, hence persuading herself that she, too, is able to carry out the task. Verbal persuasion is the belief that they are able to cope with baby care through encouragement from others. Emotional arousal is the psychological state of mind attributed with performing a task. Bandura's self-efficacy theory postulates that one's self-efficacy operates to reduce feelings of stress and depression and is crucial in determining one's emotional reaction toward a situation.

The amount of social support received and the level of maternal self-efficacy have an impact on a mother's emotional well-being. Inconsistent or low levels of social support have been found to be strongly associated with PND [34]. A study conducted in Canada reported the importance of social support in reducing PND [37]. Other research studies have also found that poor self-efficacy among mothers and a lack of social support are strongly associated with PND [21,38-41]. These studies support that self-efficacy, social support, and PND are associated variables, which are important in determining maternal outcomes. However, only one study has evaluated their relationships in first-time mothers, which confirmed their interrelated relationship and that all three components should be addressed [40].

\section{Home-Based Psychoeducation Intervention}

To combat psychological stressors from parenting, psychoeducational programs aim to address knowledge deficit regarding the postnatal period and build on the participants' strengths and resources to promote emotional coping and parenting skills development [42]. Several studies have shown that psychoeducation programs have been one of the crucial interventions to enhance maternal self-efficacy antenatally and postnatally to improve maternal outcomes [43-45]. In 2012, a preliminary study was conducted in Singapore, which reported self-efficacy improvement and PND reduction in mothers receiving a home-based psychoeducational intervention [46]. Another study exploring women's experiences of midwifery home visits revealed that women felt vulnerable in the early postpartum period but also felt that having a personal relationship with a midwife was important [47]. However, with the shortage of midwives and nurses, a home-based intervention might not be the most accessible and cost-effective method of delivering care.

\section{Accessible and Cost-Effective Web-Based Psychoeducational Intervention}

A Web-based intervention might be able to address the above problem by increasing accessibility and reducing costs associated with home visits without compromising the effectiveness of a psychoeducational intervention on maternal outcomes. A meta-analysis reported the effectiveness of a Web-based intervention in achieving positive outcomes for individuals [48]. Moreover, studies have shown that socioeconomic status, including low education and low income, increases the risk of first-time mothers developing depressive symptoms [49]. Therefore, examining the cost-effectiveness of a Web-based psychoeducational intervention may help to keep costs to a minimum and to make it affordable to all mothers regardless of socioeconomic status while achieving positive maternal outcomes. Moreover, in Singapore, the Home Access Program allows lower income households to be able to afford fiber broadband connectivity and a tablet at a subsidized rate, thereby increasing accessibility to resources online [50].

\section{Theoretical Framework}

The theoretical framework adopted for this study follows Bandura's self-efficacy theory [36], the concepts of social support including functional and structural support that can ease a smooth progression to motherhood [40,51], as well as the interrelationships between self-efficacy, social support, and emotional well-being [52-54]. The framework used in this study is similar to the one used in our preliminary study [46]. The psychoeducation intervention programs, both Web-based and home-based, are developed to promote maternal self-efficacy, social support, and mother's psychological well-being.

\section{Aims}

This study aims to evaluate the effectiveness and cost-effectiveness of Web-based and home-based postnatal psychoeducational programs for first-time mothers on maternal outcomes.

\section{Hypotheses}

The hypotheses are as follows:

1. When compared with mothers in the control group, mothers in Web-based and home-based psychoeducational intervention groups will report a significantly:

- higher level of self-efficacy,

- higher level of social support received,

- lower level of anxiety and depression, and

- higher level of satisfaction with postnatal services.

2. When compared with those in the home-based psychoeducational intervention group, mothers in the Web-based psychoeducational intervention group will not report significantly poorer aforementioned maternal outcomes.

3. It is more cost-effective to provide a Web-based psychoeducational intervention than a home-based psychoeducational intervention and routine care. 


\section{Methods}

\section{Design}

A randomized controlled three-group experimental design was used. First-time mothers $(n=204)$ were recruited from a public hospital in Singapore. Recruited mothers were randomly assigned into any of the three groups: intervention group 1 (receiving the Web-based psychoeducational intervention plus routine care), intervention group 2 (receiving the home-based psychoeducational intervention plus routine care), and the control group (receiving routine care). Research assistant (RA2) who helped with follow-up data collection did not have any knowledge of the treatment allocation of participants, which was completed by another research assistant (RA1).

\section{Participants}

The criteria for inclusion were first-time mothers who: (1) had full-term pregnancy, (2) were 21 years old and older, (3) were able to read and speak in English, (4) had Internet access through a computer or a smartphone, and (5) planned to reside in Singapore for the 6 months post delivery. The exclusion criteria were mothers who: (1) had identified physical or mental disorders (eg, cognitive impairment, schizophrenia, psychosis, or suicidal signs) before and during pregnancy that would hinder their ability to participate in the study, (2) had complicated assisted delivery with 4th degree perineal tear, and/or (3) gave birth to a stillborn child or a child with congenital anomalies or medical complications (eg, pathological jaundice), which required specialized attention in the hospital. A group of participants (15 from each group) were selected to participate in the process evaluation for the purpose of obtaining their opinions and comments on the Web-based, home-based psychoeducational intervention, and routine care.

\section{Web-Based and Home-Based Psychoeducational Intervention}

The Web-based and home-based interventions were developed based on Bandura's theory on self-efficacy [55], social support, findings from previous literature [38-40,43], and local preliminary studies [40,46,55-61]. Both interventions contain similar intervention contents but with different modes of delivery-Web-based or home visits. Table 1 shows the comparison of protocol of routine care, and Web-based and home-based postnatal psychoeducational interventions.

\section{Web-Based Psychoeducational Intervention}

The mothers assigned to intervention group 1 had participants receiving periodic care and a Web-based postnatal psychoeducational intervention with 1-month access. The intervention has been summarized in Table 1 . The videos were developed based on current practices in local hospitals. There was a peer discussion forum where the participant could communicate with other participants, or a confidential corner for participants to ask personal questions. Expert advice was also provided by RA2 and other team members when needed. There were also thrice-weekly telephone reminders, which lasted for about $3 \mathrm{~min}$ each, solely as a reminder for participants to assess the website without any additional education provided.

Table 1. Comparison of protocol of routine care, and Web-based and home-based postnatal psychoeducational interventions.

\begin{tabular}{|c|c|c|}
\hline Control group: routine care only & $\begin{array}{l}\text { Intervention group } 1 \text { : Web-based intervention } \\
\text { (+ routine care) }\end{array}$ & $\begin{array}{l}\text { Intervention group } 2 \text { : home-based intervention } \\
(+ \text { routine care })\end{array}$ \\
\hline \multirow[t]{2}{*}{$\begin{array}{l}\text { Before discharge: Routine education received in } \\
\text { the hospital }\end{array}$} & $\begin{array}{l}\text { Before discharge: Access to website information, } \\
\text { and Web-based audio and video materials }\end{array}$ & Before discharge: Provision of booklet \\
\hline & $\begin{array}{l}\text { Main content covered in the website, audios, and } \\
\text { videos, which included the following: postnatal } \\
\text { experiences; maternal self-care (including } \\
\text { physical, emotional, and sexual health); newborn } \\
\text { care, and social support }\end{array}$ & $\begin{array}{l}\text { Main content covered in the booklet included } \\
\text { the following: postnatal experiences; maternal } \\
\text { self-care (including physical, emotional, and } \\
\text { sexual health); newborn care, and social support }\end{array}$ \\
\hline
\end{tabular}

At 2 weeks post-delivery: Follow-up with obstetrician; examination of episiotomy or cesarean wound; wound dressing for cesarean wound; and use of Edinburgh Postnatal Depression Scale (EPDS) 1st week after discharge: Go through website information, and Web-based audio and video materials

Peer discussion forum, and a confidential corner for personal questions and expert advice

Mothers can discuss their concerns related to self-care and newborn care; expert (RA2 and other team members) will access the website and respond to the questions raised daily

At 6 weeks post-delivery: Follow-up with the obstetrician; advice on: breastfeeding; PAP smear; intrauterine contraceptive device upon patients' request; and EPDS for those mothers who have defaulted the 2 weeks appointment 2nd to 4th week after discharge: Weekly telephone calls $x 3$; reinforce the use of website resources 1st week after discharge: One hour face-to-face education at home

Main content covered in the face-to-face session is the same as in the booklet

2nd to 4th week after discharge: Weekly telephone calls x 3; reinforcement of the content covered during home visit; find out new challenges faced by the mothers; and provide individualized support as per mothers' needs 


\section{Home-Based Psychoeducational Intervention}

Participants assigned to intervention group 2 received periodic care and a home-based postnatal psychoeducational intervention. There was a 1-hour face-to-face psychoeducation via a home visit by a registered nurse (RA1). An educational booklet was also developed based on the one used in the preliminary study [61] with new evidence-based knowledge added, such as mindfulness-based practice and using cold cabbage leaves for managing breast engorgement [61], which were provided to participants before hospital discharge. The main contents in the booklet provided for the home-based intervention were identical to the one from group 1 . The contents were validated by an expert panel, consisting of 5 health care professionals (2 professors in Obstetric and Gynecological Nursing, 1 senior consultant in obstetrics, 1 experienced midwife in postnatal care, and 1 postnatal psychologist). There was also a thrice-weekly telephone follow-up to answer mothers' queries.

\section{Control Group}

Participants assigned to the control group only received the routine care provided by the hospital.

\section{Sample Size Determination}

In similar studies, psychosocial and educational interventions have resulted in a medium effect size on outcomes similar to this study $[44,52,62]$; hence, the study intervention was regarded to have a medium size effect on outcome variables. On the basis of a power analysis, to achieve a medium effect size of 0.55 , using a power of $80 \%$ and a significance level of 5\% (2-sided), 52 participants would be needed in each group [63]. Although a dropout rate of $30 \%$ was reported by a previous study [52] and our preliminary study [46], we anticipated a lower dropout rate of $24 \%$ in this study because of better communication channels established with participants (eg, using social media such as WhatsApp to remind participants, follow-up questionnaire conducted through a Web-based survey). Therefore, a minimum sample of $204(52 \times 3 / 0.76)$ participants with 68 in each group was needed. For process evaluation, a purposive sampling of 45 participants (15 from each group) with different posttest 1 self-efficacy scores ( 5 high, 5 moderate, and 5 low scores from each group) were invited to participate in a process evaluation interviews, or until data saturation was achieved.

\section{Randomization}

A random sequence generator was used to generate three sets with 68 unique random integers in each set that ranged from 1 to 204 without being sorted [64]. Three sets of numbers were randomly assigned for intervention group 1, intervention group 2 , or control group, respectively. The blinding of the participant was not possible because of the nature of the intervention. All 204 unique numbers indicating the group were placed in an opaque box. After assessing eligibility and obtaining informed consent, participants were assigned randomly to either of the groups by drawing a number from the box. Participants were reminded that there will be no change of groups after numbers are drawn.

\section{Outcome Measures and Instruments}

The outcome measured included are maternal parental self-efficacy (MPSE), social support, PND, anxiety, satisfaction with postnatal care, and cost related to health care services used postnatally. The instruments used to measure the key variables included Perceived Maternal Parental Self-efficacy (PMPSE), the modified Perinatal Infant Care Social Support (PICSS-modified), Edinburgh Postnatal Depression Scale (EPDS), Anxiety subscale of the Hospital Anxiety and Depression Scale (HADS-A), Ordinal Descriptive Scale (ODS), and Questionnaire on Healthcare Service Utilization (QHSU).

\section{Perceived Maternal Parental Self-Efficacy}

The PMPSE is a widely used 17-item instrument that measures maternal self-efficacy in the postpartum period. Each item is rated on a four-point Likert scale. The Cronbach alpha values in a previous study and a local study were .91 [65] and .92, respectively [54].

\section{Social Support}

The modified PICSS includes the Functional Social Support Measuring Subscale (FSSMS) and Structural Social Support Measuring Subscale (SSSMS). The FSSMS is a 16-item subscale used to measure maternal perceived social support [57] with Cronbach alpha values of .80 in a previous study [57] and .76 in a local study [54]. The SSSMS is a 6-item subscale used to measure the structural dimension of an individual's social network with a Cronbach alpha of .83 in a local study [54].

\section{Psychological Well-Being}

The EPDS and HADS-A were used to measure mothers' psychological well-being, including PND and anxiety. The 10-item EPDS has been used to measure PND widely. The recommended cutoff for probable cases of PND is 12 or 13 [66]. The sensitivity and specificity of the EPDS were $68 \%$ to $80 \%$ and $77 \%$, respectively, and the Cronbach alpha was .88 [67]. The HADS-A is a 7-item instrument that is most commonly used to assess anxiety symptoms [68]. It is rated on a 4-point scale of 0 to 3 . The minimum score of 8 determines the presence of anxiety symptoms [69]. The sensitivity and specificity of HADS-A were $93 \%$ and $90 \%$, respectively, when it was used for pregnant women from Nigeria [70].

\section{Satisfaction With Postnatal Care}

The ODS is a 6-point Likert scale for assessing mothers' satisfaction with the postnatal care they received [46,59].

\section{Health Care Service Utilization}

The QHSU was used to capture costs related to health care service usage by the mothers postnatally due to maternal- or infant-related health issues at 1 month, 3 months, and 6 months post delivery. The measurement period for the study was from entry into the program until 6 months. The questionnaire was designed by health economy experts from the Singapore Clinical Research Institute.

\section{Process Evaluation}

Semistructured interviews were conducted for exploring the information regarding the strengths, weaknesses, and perceived 
effects of the interventions, and the mothers' opinions on the current routine postnatal care provided by the hospital.

\section{Study Procedure}

Data collection commenced after the ethical approval had been obtained. The nurse in charge was contacted to determine the physical and psychological well-being of potential participants. After confirming the inclusion criteria, the participants were approached at the postnatal inpatient wards. Only after obtaining written consent would the mother be recruited into the study. This was followed by a collection of demographic data and baseline data via a self-administered questionnaire before randomization takes place. Outcomes were measured at the following time points for all participants in all three groups: (1) after delivery and before discharge (baseline data), (2) immediately after the intervention (posttest one, at the end of 1 month post delivery), (3) 2 months after the intervention (posttest 2, 3 months post delivery), and (4) 5 months after the intervention (posttest 3, 6 months post delivery).

RA1 made a visit to the hospital regularly to recruit participants and was responsible for informing all participants of their allocated group, and collection of baseline data for all participants. RA1 provided participants in the Web-based intervention group with Web access (individual username and password) for 1 month. For participants assigned to the home-based intervention group, RA1 provided the educational booklet before discharge and arranging home visits at about 5 to 10 days post delivery, as this is the most crucial period for mothers, as well as 3 telephone calls [71].

RA2 was responsible for the Web-based postnatal psychoeducational intervention (eg, providing expert advice in group discussion forums and confidential corner, and 3 reminder phone calls). RA2 was responsible for the collection of all posttest data, including process evaluation interviews.

\section{Data Analysis}

Quantitative data will be analyzed using IBM SPSS version 24 for Windows (IBM Corp). Descriptive statistics such as mean, standard deviation, and range will be used to report continuous data, whereas frequency and percentages will be used for the nominal and ordinal data. Inferential statistics such as ANOVA will be used to analyze the differences of baseline outcomes among the three groups, whereas chi-square tests will be used to compare the sociodemographics among the three groups.

To answer the hypothesis 1, repeated measures ANCOVA, adjusted for confounding variables (eg, age, ethnicity), will be used to test the effects of both interventions on maternal self-efficacy, social support, postnatal depression, and anxiety across four time points. Univariate ANCOVA will be used to test the difference of each of the three outcomes among three groups at each posttest time point by adjusting for confounding factors (eg, baseline data, age, and ethnicity). Chi-square test or Poisson regression will be used to determine maternal satisfaction with the postnatal supportive care at each posttest time points. To answer hypothesis 2, a 95\% CI of the mean difference of maternal self-efficacy scores will be calculated. If the lower boundary is more than -5 , the hypothesis will be accepted. To address hypothesis 3, we will calculate the base case incremental cost-effectiveness ratios (ICER) of the two intervention groups compared with the control group. We captured the 6-month costs at three posttest time points: (1) health care system perspectives, including service providers that mothers consumed because of maternal and/or neonatal related conditions such as GP clinic, polyclinic, private clinic, hospital out-patient, hospital Accident and Emergency and (2) participant's out-of-pocket cost of transportation fee for these visits. In addition, fixed costs (also considered health care system perspectives) such as development and implementation of Web-based intervention, delivery of home-based intervention, and training of RAs were also captured. The effectiveness of the intervention on four outcomes (namely self-efficacy, social support, depression and anxiety) will be calculated. The ICERs for each outcome will be plotted in the cost-effectiveness plane to examine the dominance and extended dominance, and then the cost-effectiveness acceptability curves will be generated to demonstrate the cost-effectiveness of the alternatives using difference willingness-to-pay thresholds [72-78].

Qualitative data from the interviews will be analyzed using thematic analysis [79-83]. The audiotaped interview data will be transcribed verbatim by RA2 concurrently with the data collection to capture nonverbal nuances. Transcribed data will be color coded first, which will then be collated to form subthemes, and eventually to form themes [84]. Two investigators will be involved in the analysis process to compare and discuss the codes, subthemes, and themes that are generated and to achieve consensus. Rigor, including credibility, transferability, dependability, and confirmability, will be considered carefully in the study process [85].

\section{Ethical Considerations}

Ethics approval for the study was received from the relevant ethics review board before commencing the study (Ref: 2015/01189) on 25 January 2016. A participant information sheet, which provides a brief introduction to the study as well as the benefits and risks of their participation in the study was explained. Anonymity and voluntary participation was ensured. Each participant's written consent was also obtained, including consent for audio recording during the process evaluation interview. Participants whose EPDS scores were more than 13 were referred to their obstetrician for further follow-up. A token of appreciation was provided to all participants for their time spent to participate in the study and this is a common practice in the study country.

\section{Results}

The booklet for the home-based intervention group and the website for the Web-based intervention (including the audio and video files) have been developed. The recruitment of participants started in October 2016 and was completed in February 2017 with 68 mothers in each group $(n=204)$. All follow-up data were collected by August 2017. The consolidated standards of reporting trial flowchart can be found in Figure 1. The projected timeline for completion of data entry and data analysis is around March to June 2018. 
Figure 1. Consolidated standards of the reporting trial flowchart of the study. PMPSE: Perceived maternal parental self-efficacy; PICSS-modified: Modified Perinatal Infant Care Social Support Scale; EPDS: Edinburg Postnatal Depression Scale; HADS-A: Hospital Anxiety and Depression Scale (Anxiety Subscale); ODS: Ordinal Descriptive Scale (satisfaction with postnatal care); QHSU: Questionnaire on Healthcare Services Utilization.

$$
\text { Assessed for eligibility (n...) }
$$

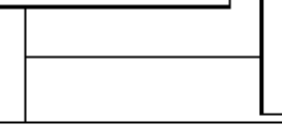

$$
\text { Excluded (n...) }
$$

-Not meeting the inclusion

criteria $(\mathrm{n} . .$.

\begin{tabular}{|c|c|c|}
\hline \multicolumn{3}{|c|}{$\begin{array}{l}\text { Baseline measurements (after delivery, before di scharge): } \\
\text { PMPSE, PICSS-modified, EPDS, HADS-A, Demographic and Newborn data } \\
\text { Randomization }\end{array}$} \\
\hline $\begin{array}{l}\text { Intervention group } \\
1(\mathrm{n}=68) \\
\text { Web-based postnatal } \\
\text { psychoeducati onal } \\
\text { intervention+ routing } \\
\text { care Did not receive } \\
\text { intervention, lost to } \\
\text { follow-up, etc. (n...) }\end{array}$ & $\begin{array}{l}\text { Intervention group } \\
2 \text { (n=68) } \\
\text { Home-based postnatal } \\
\text { psychoeducational } \\
\text { interventi on+ routing } \\
\text { care Did not receive } \\
\text { intervention, lost to } \\
\text { follow-up, etc. (n...) }\end{array}$ & $\begin{array}{l}\text { Control group } \\
(\mathrm{n}=68) \\
\text { Routine care } \\
\text { Did not receive } \\
\text { intervention, lost to } \\
\text { follow-up, etc. (n...) }\end{array}$ \\
\hline \multicolumn{3}{|c|}{ Completed trial (n...) } \\
\hline \multicolumn{3}{|c|}{$\begin{array}{l}\text { Posttest I data collection: Immediately after intervention ( } 1 \text { month post delivery) } \\
\text { PMPSE, PICSS-modified, EPDS, HADS-A, ODS, QHSU, process evaluation }\end{array}$} \\
\hline \multicolumn{3}{|c|}{$\begin{array}{l}\text { Posttest } 2 \text { data collection: } 2 \text { months after intervention ( } 3 \text { months post delivery) } \\
\text { PMPSE, PICSS-modified, EPDS, HADS-A, ODS, QHSU }\end{array}$} \\
\hline \multicolumn{3}{|c|}{$\begin{array}{c}\text { Posttest } 3 \text { data collection: } 5 \text { months after intervention ( } 6 \text { months post delivery) } \\
\text { PMPSE, PICSS-modified, EPDS, HADS-A, ODS, QHSU }\end{array}$} \\
\hline
\end{tabular}

-Refused to participate (n...)

\section{Discussion}

\section{Principal Findings}

This proposed study is based on previous studies conducted by the same research team $[22,46,59,60]$. With minimal time spent from midwives and/or nurses, this study will provide empirical support and identify a clinically useful and potentially effective and cost-effective Web-based postnatal psychoeducational program in promoting maternal self-efficacy, seeking social support from health care professionals, and family members), and psychological well-being by equipping mothers with knowledge, skills, and support in the postnatal period. The Web-based psychoeducational intervention will reduce the need for face-to-face home-based interventions. If the proposed Web-based psychoeducational intervention is as effective and more cost-effective when compared with the home-based intervention, it will be introduced to the hospital policy maker and be adopted as a standard care to promote the quality of postnatal care for the new mothers and increase mothers' satisfaction with postnatal services, without needing extra manpower to provide postnatal home visits to mothers.

The short term clinical implication of this study is such that a comprehensive and well-designed Web-based postnatal psychoeducational program will be developed for first-time mothers. Following that, an understanding will be established on the determinants of the outcome variables in improving maternal outcomes. These strategies can also be incorporated into the services provided by midwifery-led clinics.

In the long term, the practicality of both interventions will be determined. If the Web-based intervention is reported to be more practical, effective, and cost-effective as compared with 
the home-based intervention and the current standard routine care, the learning package adopted in the Web-based intervention can then be provided to first-time mothers to address any doubts and questions. With the implementation of the Web-based intervention, first-time mothers will then be able to effectively allocate their time and resources when it comes to the usage of emergency medical services in addressing minor issues related to maternal and newborn care. Health care professionals will also be able to effectively allocate appropriate resources when it comes to educating new mothers and providing consultations to address queries from mothers. The Web-based intervention can also be beneficial to mothers as it provides continuity of care for new mothers after their discharge from the hospital and enhances the safety of both the mother and the newborn. Empowering mothers with confidence back at home contributes to the overall well-being of mothers and newborns [46]. A positive postnatal experience could also potentially influence future birth plans and eventually contribute to an increase fertility rate in Singapore. In addition, mothers in countries other than Singapore can also benefit from the website resources.

\section{Limitations of the Study}

One of the limitations was the inability to blind the participants from the intervention because of the nature of the interventions. This study was only conducted in a single tertiary hospital in Singapore and all participating mothers were English-speaking mothers. The results tested on mothers from the private hospitals and mothers who speak other languages might be different.

\section{Conclusions}

This randomized controlled trial will provide empirical evidence to support the effectiveness and cost-effectiveness of the Web-based and home-based psychoeducational interventions in promoting maternal outcomes. This study also has potential benefits in reducing health care costs associated with postnatal supportive services. Future studies should include non-English-speaking mothers and mothers from other maternity hospitals in Singapore and other countries to enhance the generalizability of the study findings.

\section{Acknowledgments}

This study received the National Medical Research Council Health Services Research Competitive Research Grant from the Ministry of Health, Singapore (Project No.: NMRC/HSRG/0051/2015). The authors appreciate the Medical Publications Support Unit of the National University Health System, Singapore, for the assistance provided in the language editing of this manuscript.

\section{Conflicts of Interest}

None declared.

\section{Multimedia Appendix 1}

Original peer-review reports.

[PDF File (Adobe PDF File), 2MB-Multimedia Appendix 1]

\section{References}

1. Cowan CP, Cowan PA. When Partners Become Parents: The Big Life Change for Couples. London: Lawrence Erlbaum Associates Publishers; 2000.

2. Hammes T, Sebold LF, Kempfer SS, Girondi JB. Nursing care in postpartum adaptation: perceptions of Brazilian mothers. J Nurs Educ Pract 2014;4(12):125-133. [doi: 10.5430/jnep.v4n12p125]

3. Harwood K, McLean N, Durkin K. First-time mothers' expectations of parenthood: what happens when optimistic expectations are not matched by later experiences? Dev Psychol 2007 Jan;43(1):1-12. [doi: 10.1037/0012-1649.43.1.1] [Medline: $\underline{17201504]}$

4. Rubin R. Attainment of the maternal role: Part I. Processes. Nurs Res 1967;16(3):237-245.

5. Forster DA, McLachlan HL, Rayner J, Yelland J, Gold L, Rayner S. The early postnatal period: exploring women's views, expectations and experiences of care using focus groups in Victoria, Australia. BMC Pregnancy Childbirth 2008 Jul 22;8:27 [FREE Full text] [doi: 10.1186/1471-2393-8-27] [Medline: 18644157$]$

6. Murray L, Arteche A, Fearon P, Halligan S, Goodyer I, Cooper P. Maternal postnatal depression and the development of depression in offspring up to 16 years of age. J Am Acad Child Adolesc Psychiatry 2011 May;50(5):460-470. [doi: 10.1016/j.jaac.2011.02.001] [Medline: 21515195]

7. Healthhub.sg. Healthy start for your pregnancy URL: https://www.healthhub.sg/sites/assets/Assets/PDFs/HPB/ Healthy\%20Start\%20For\%20Your\%20Pregnancy/Healthy\%20Start\%20for\%20your\%20Pregnancy.pdf [WebCite Cache ID 6q5Ljd2Xn]

8. Hatem M, Sandall J, Devane D, Soltani H, Gates S. Midwife-led versus other models of care for childbearing women. Cochrane Database Syst Rev 2008 Oct 8(4):CD004667. [doi: 10.1002/14651858.CD004667.pub2] [Medline: 18843666]

9. Dahlen HG, Barclay LM, Homer CS. The novice birthing: theorising first-time mothers' experiences of birth at home and in hospital in Australia. Midwifery 2010 Feb;26(1):53-63. [doi: 10.1016/j.midw.2008.01.012] [Medline: 18394766] 
10. Fenwick J, Butt J, Dhaliwal S, Hauck Y, Schmied V. Western Australian women's perceptions of the style and quality of midwifery postnatal care in hospital and at home. Women Birth 2010 Mar;23(1):10-21. [doi: 10.1016/j.wombi.2009.06.001] [Medline: 19632912]

11. Institute of Medicine. Prenatal Care: Reaching Mothers, Reaching Infants. Washington, DC: The National Academies Press; 1988.

12. Christie J, Bunting B. The effect of health visitors' postpartum home visit frequency on first-time mothers: cluster randomised trial. Int J Nurs Stud 2011 Jun;48(6):689-702. [doi: 10.1016/j.ijnurstu.2010.10.011] [Medline: 21129744]

13. Moh. 2017. Admission for normal (vaginal) delivery URL: https:/www.moh.gov.sg/content/moh web/home/ costs and financing/hospital-charges/Total-Hospital-Bills-By-condition-procedure/Childbirth Delivery Normal.html [accessed 2018-01-16] [WebCite Cache ID 6wVRjdI3c]

14. Hung CH, Yu CY, Chang SJ, Stocker J. Postpartum psychosocial changes among experienced and inexperienced mothers in Taiwan. J Transcult Nurs 2011 Jul;22(3):217-224. [doi: 10.1177/1043659611404432] [Medline: 21519063]

15. Bowman KG. Postpartum learning needs. J Obstet Gynecol Neonatal Nurs 2005;34(4):438-443. [doi: 10.1177/0884217505276054] [Medline: 16020411]

16. Diagnostic and Statistical Manual of Mental Disorders (DSM-5®), Fifth Edition. Arlington, VA: American Psychiatric Association Publishing; 2013.

17. Woolhouse H, Gartland D, Perlen S, Donath S, Brown SJ. Physical health after childbirth and maternal depression in the first 12 months post partum: results of an Australian nulliparous pregnancy cohort study. Midwifery 2014 Mar;30(3):378-384. [doi: 10.1016/j.midw.2013.03.006] [Medline: 23619027]

18. O'hara MW, Swain AM. Rates and risk of postpartum depression-a meta-analysis. Int Rev Psychiatry 1996;8(1):37-54. [doi: 10.3109/09540269609037816]

19. Falah-Hassani K, Shiri R, Vigod S, Dennis CL. Prevalence of postpartum depression among immigrant women: a systematic review and meta-analysis. J Psychiatr Res 2015 Nov;70:67-82. [doi: 10.1016/j.jpsychires.2015.08.010] [Medline: 26424425]

20. Grekin R, O'Hara MW. Prevalence and risk factors of postpartum posttraumatic stress disorder: a meta-analysis. Clin Psychol Rev 2014 Jul;34(5):389-401. [doi: 10.1016/j.cpr.2014.05.003] [Medline: 24952134]

21. Chee CY, Lee DT, Chong YS, Tan LK, Ng TP, Fones CS. Confinement and other psychosocial factors in perinatal depression: a transcultural study in Singapore. J Affect Disord 2005 Dec;89(1-3):157-166. [doi: 10.1016/j.jad.2005.09.004] [Medline: 16257451]

22. Ong SF, Chan WS, Shorey S, Chong YS, Klainin-Yobas P, He HG. Postnatal experiences and support needs of first-time mothers in Singapore: a descriptive qualitative study. Midwifery 2014 Jun;30(6):772-778. [doi: 10.1016/j.midw.2013.09.004] [Medline: 24161493]

23. Habel C, Feeley N, Hayton B, Bell L, Zelkowitz P. Causes of women's postpartum depression symptoms: men's and women's perceptions. Midwifery 2015 Jul;31(7):728-734. [doi: 10.1016/j.midw.2015.03.007] [Medline: 25921442]

24. Meyer JH. Neurochemical Imaging and Depressive Behaviours. In: Behavioral Neurobiology of Depression and Its Treatment. Berlin, Heidelberg: Springer-Verlag; 2013.

25. O'hara MW. Post-partum 'blues,' depression, and psychosis: a review. J Psychosom Obstet Gynaecol 1987;7(3):205-227. [doi: 10.3109/01674828709040280]

26. Demontigny F, Girard ME, Lacharité C, Dubeau D, Devault A. Psychosocial factors associated with paternal postnatal depression. J Affect Disord 2013 Aug 15;150(1):44-49. [doi: 10.1016/j.jad.2013.01.048] [Medline: 23489392]

27. Letourneau NL, Dennis CL, Benzies K, Duffett-Leger L, Stewart M, Tryphonopoulos PD, et al. Postpartum depression is a family affair: addressing the impact on mothers, fathers, and children. Issues Ment Health Nurs 2012 Jul;33(7):445-457. [doi: 10.3109/01612840.2012.673054] [Medline: 22757597]

28. Schachman KA, Lee RK, Lederma RP. Baby boot camp: facilitating maternal role adaptation among military wives. Nurs Res 2004;53(2):107-115. [Medline: 15084995]

29. Leahy-Warren P, McCarthy G. Postnatal depression: prevalence, mothers' perspectives, and treatments. Arch Psychiatr Nurs 2007 Apr;21(2):91-100. [doi: 10.1016/j.apnu.2006.10.006] [Medline: 17397691]

30. Sampson M, Villarreal Y, Padilla Y. Association between support and maternal stress at one year postpartum: does type matter? Soc Work Res 2015 Mar;39(1):49-60. [doi: 10.1093/swr/svu031]

31. Huang CY, Costeines J, Ayala C, Kaufman JS. Parenting stress, social support, and depression for ethnic minority adolescent mothers: impact on child development. J Child Fam Stud 2014 Feb;23(2):255-262 [FREE Full text] [doi:

10.1007/s10826-013-9807-1] [Medline: 24653641]

32. Reid KM, Taylor MG. Social support, stress, and maternal postpartum depression: a comparison of supportive relationships. Soc Sci Res 2015 Nov;54:246-262. [doi: 10.1016/j.ssresearch.2015.08.009] [Medline: 26463547]

33. Bäckström C, Larsson T, Wahlgren E, Golsäter M, Mårtensson LB, Thorstensson S. 'It makes you feel like you are not alone': Expectant first-time mothers' experiences of social support within the social network, when preparing for childbirth and parenting. Sex Reprod Healthc 2017 Jun;12:51-57 [FREE Full text] [doi: 10.1016/j.srhc.2017.02.007] [Medline: 28477932] 
34. Negron R, Martin A, Almog M, Balbierz A, Howell EA. Social support during the postpartum period: mothers' views on needs, expectations, and mobilization of support. Matern Child Health J 2013 May;17(4):616-623 [FREE Full text] [doi: 10.1007/s10995-012-1037-4] [Medline: 22581378]

35. Gross D, Rocissano L. Maternal confidence in toddlerhood: its measurement for clinical practice and research. Nurse Pract 1988 Mar;13(3):19-22, 25, 28-9. [Medline: 3374866]

36. Bandura A. Self-efficacy: toward a unifying theory of behavioral change. Psychol Rev 1977 Mar;84(2):191-215. [Medline: 847061]

37. Dennis CL, Hodnett E, Kenton L, Weston J, Zupancic J, Stewart DE, et al. Effect of peer support on prevention of postnatal depression among high risk women: multisite randomised controlled trial. BMJ 2009 Jan 15;338:a3064 [FREE Full text] [Medline: 19147637]

38. Azmoude E, Jafarnejade F, Mazlom S. The predictors for maternal self-efficacy in early parenthood. JMRH 2015 Apr;3(2):368-376. [doi: 10.22038/jmrh.2015.4050]

39. Kohlhoff J, Barnett B. Parenting self-efficacy: links with maternal depression, infant behaviour and adult attachment. Early Hum Dev 2013 Apr;89(4):249-256. [doi: 10.1016/j.earlhumdev.2013.01.008] [Medline: 23398731]

40. Leahy-Warren P, McCarthy G, Corcoran P. First-time mothers: social support, maternal parental self-efficacy and postnatal depression. J Clin Nurs 2012 Feb;21(3-4):388-397. [doi: 10.1111/j.1365-2702.2011.03701.x] [Medline: 21435059]

41. Zubaran C, Foresti K. The correlation between breastfeeding self-efficacy and maternal postpartum depression in southern Brazil. Sex Reprod Healthc 2013 Mar;4(1):9-15. [doi: 10.1016/j.srhc.2012.12.001] [Medline: 23427927]

42. Rowe HJ, Fisher JR. Development of a universal psycho-educational intervention to prevent common postpartum mental disorders in primiparous women: a multiple method approach. BMC Public Health 2010 Aug 18;10:499 [FREE Full text] [doi: 10.1186/1471-2458-10-499] [Medline: 20718991]

43. Ngai FW, Wai-Chi Chan S, Ip WY. Predictors and correlates of maternal role competence and satisfaction. Nurs Res 2010;59(3):185-193. [doi: 10.1097/NNR.0b013e3181dbb9ee] [Medline: 20404775]

44. Ip WY, Tang CS, Goggins WB. An educational intervention to improve women's ability to cope with childbirth. J Clin Nurs 2009 Aug;18(15):2125-2135. [doi: 10.1111/j.1365-2702.2008.02720.x] [Medline: 19583645]

45. Dennis CL. Identifying predictors of breastfeeding self-efficacy in the immediate postpartum period. Res Nurs Health 2006 Aug;29(4):256-268. [doi: 10.1002/nur.20140] [Medline: 16847899]

46. Shorey S, Chan SW, Chong YS, He HG. A randomized controlled trial of the effectiveness of a postnatal psychoeducation programme on self-efficacy, social support and postnatal depression among primiparas. J Adv Nurs 2015 Jun;71(6):1260-1273. [doi: 10.1111/jan.12590] [Medline: 25496615]

47. Dahlberg U, Haugan G, Aune I. Women's experiences of home visits by midwives in the early postnatal period. Midwifery 2016 Aug;39:57-62. [doi: 10.1016/j.midw.2016.05.003] [Medline: 27321721]

48. Wantland DJ, Portillo CJ, Holzemer WL, Slaughter R, McGhee EM. The effectiveness of Web-based vs. non-Web-based interventions: a meta-analysis of behavioral change outcomes. J Med Internet Res 2004 Nov 10;6(4):e40 [FREE Full text] [doi: 10.2196/jmir.6.4.e40] [Medline: 15631964$]$

49. Goyal D, Gay C, Lee KA. How much does low socioeconomic status increase the risk of prenatal and postpartum depressive symptoms in first-time mothers? Womens Health Issues 2010;20(2):96-104 [FREE Full text] [doi: 10.1016/j.whi.2009.11.003] [Medline: 20133153]

50. Imda. 2017. Home Access Programme to Benefit 8,000 Low Income Households URL: https://www.imda.gov.sg/about/ newsroom/archived/ida/media-releases/2015/home-access-programme-to-benefit-8000-low-income-households[WebCite Cache ID 6ude91COz]

51. Wilkins C. A qualitative study exploring the support needs of first-time mothers on their journey towards intuitive parenting. Midwifery 2006 Jun;22(2):169-180. [doi: 10.1016/j.midw.2005.07.001] [Medline: 16356610]

52. Ngai FW, Chan SW, Ip WY. The effects of a childbirth psychoeducation program on learned resourcefulness, maternal role competence and perinatal depression: a quasi-experiment. Int J Nurs Stud 2009 Oct;46(10):1298-1306. [doi: 10.1016/j.ijnurstu.2009.03.007] [Medline: 19361801]

53. Haslam DM, Pakenham KI, Smith A. Social support and postpartum depressive symptomatology: the mediating role of maternal self-efficacy. Infant Ment Health J 2006 May;27(3):276-291. [doi: 10.1002/imhj.20092]

54. Shorey S, Chan SW, Chong YS, He HG. Maternal parental self-efficacy in newborn care and social support needs in Singapore: a correlational study. J Clin Nurs 2014 Aug;23(15-16):2272-2282. [doi: 10.1111/jocn.12507] [Medline: 24372630]

55. Bandura A. Self-Efficacy: The Exercise of Control. New York: Freeman; 1997.

56. Brown S, Lumley J. Physical health problems after childbirth and maternal depression at six to seven months postpartum. BJOG 2000 Oct;107(10):1194-1201. [doi: 10.1111/j.1471-0528.2000.tb11607.x]

57. Johansson K, Darj E. What type of information do parents need after being discharged directly from the delivery ward? Ups J Med Sci 2004;109(3):229-238. [Medline: 15508525]

58. Kapp M. Mothers' perception of confidence with self-care and infant care. J Perinat Educ 1998;7(4):17-25. [doi: 10.1624/105812498X86864] 
59. Ong SF, Chan WS, Shorey S, Chong YS, Klainin-Yobas P, He HG. Postnatal experiences and support needs of first-time mothers in Singapore: a descriptive qualitative study. Midwifery 2014 Jun;30(6):772-778. [doi: 10.1016/j.midw.2013.09.004] [Medline: 24161493]

60. Shorey S, Chan SW, Chong YS, He HG. Perceptions of primiparas on a postnatal psychoeducation programme: the process evaluation. Midwifery 2015 Jan;31(1):155-163. [doi: 10.1016/j.midw.2014.08.001] [Medline: 25174540]

61. Shorey S, Chan W, Chong Y, He HG. The effectiveness of a postnatal psycho education programme on outcomes of first-time mothers in Singapore. National University of Singapore 2013:-.

62. Saisto T, Salmela-Aro K, Nurmi JE, Halmesmäki E. Psychosocial characteristics of women and their partners fearing vaginal childbirth. BJOG 2001 May;108(5):492-498 [FREE Full text] [Medline: 11368135$]$

63. Cohen J. Statistical power analysis. Curr Dir Psychol Sci 1992;1(3):98-101 [FREE Full text]

64. Random. Random Sequence Generator URL: https://www.random.org/sequences/ ?min=1\&max=204\&col=1\&format=html\&rnd=new [WebCite Cache ID 6qjaDyAAx]

65. Barnes CR, Adamson-Macedo EN. Perceived maternal parenting self-efficacy (PMP S-E) tool: development and validation with mothers of hospitalized preterm neonates. J Adv Nurs 2007 Dec;60(5):550-560. [doi: 10.1111/j.1365-2648.2007.04445.x] [Medline: 17973719 ]

66. Cox JL, Holden JM, Sagovsky R. Detection of postnatal depression. Development of the 10-item Edinburgh Postnatal Depression Scale. Br J Psychiatry 1987 Jun;150:782-786. [Medline: 3651732]

67. Cox JL, Chapman G, Murray D, Jones P. Validation of the Edinburgh Postnatal Depression Scale (EPDS) in non-postnatal women. J Affect Disord 1996 Jul 29;39(3):185-189. [Medline: 8856422 ]

68. Lee AM, Lam SK, Sze Mun Lau SM, Chong CS, Chui HW, Fong DY. Prevalence, course, and risk factors for antenatal anxiety and depression. Obstet Gynecol 2007 Nov;110(5):1102-1112. [doi: 10.1097/01.AOG.0000287065.59491.70] [Medline: 17978126 ]

69. Bjelland I, Dahl AA, Haug TT, Neckelmann D. The validity of the Hospital Anxiety and Depression Scale. An updated literature review. J Psychosom Res 2002 Feb;52(2):69-77. [Medline: 11832252]

70. Abiodun OA. A validity study of the Hospital Anxiety and Depression Scale in general hospital units and a community sample in Nigeria. Br J Psychiatry 1994 Nov;165(5):669-672. [Medline: 7866683]

71. Bashour HN, Kharouf MH, Abdulsalam AA, El Asmar K, Tabbaa MA, Cheikha SA. Effect of postnatal home visits on maternal/infant outcomes in Syria: a randomized controlled trial. Public Health Nurs 2008;25(2):115-125. [doi:

10.1111/j.1525-1446.2008.00688.x] [Medline: 18294180]

72. Barber JA, Thompson SG. Analysis and interpretation of cost data in randomised controlled trials: review of published studies. BMJ 1998 Oct 31;317(7167):1195-1200 [FREE Full text] [Medline: 9794854]

73. Fenwick E, Byford S. A guide to cost-effectiveness acceptability curves. Br J Psychiatry 2005 Aug;187:106-108 [FREE Full text] [doi: 10.1192/bjp.187.2.106] [Medline: 16055820]

74. Fenwick E, Claxton K, Sculpher M. Representing uncertainty: the role of cost-effectiveness acceptability curves. Health Econ 2001 Dec;10(8):779-787. [Medline: 11747057]

75. Johannesson M, Weinstein MC. On the decision rules of cost-effectiveness analysis. J Health Econ 1993;12(4):459-467. [doi: 10.1016/0167-6296(93)90005-Y]

76. Yardley L, Kirby S, Barker F, Little P, Raftery J, King D, et al. An evaluation of the cost-effectiveness of booklet-based self-management of dizziness in primary care, with and without expert telephone support. BMC Ear Nose Throat Disord 2009 Dec 29;9:13 [FREE Full text] [doi: 10.1186/1472-6815-9-13] [Medline: 20098640]

77. Chan S, Mackenzie A, Jacobs P. Cost-effectiveness analysis of case management versus a routine community care organization for patients with chronic schizophrenia. Arch Psychiatr Nurs 2000 Apr;14(2):98-104. [Medline: 10783528]

78. Drummond MF, Sculpher MJ, Claxton K, Stoddart GL, Torrance GW. Methods for the Economic Evaluation of Health Care Programmes. United Kingdom: Oxford University Press; 2015.

79. Taylor BJ, Kermode S, Roberts KL. Research in Nursing and Health Care: Creating Evidence for Practice. Australia: Thomas Nelson; 2006.

80. Braun V, Clarke V. Using thematic analysis in psychology. Qual Res Psychol 2006;3(2):77-101. [doi: 10.1191/1478088706qp063oa]

81. Bradley EH, Curry LA, Devers KJ. Qualitative data analysis for health services research: developing taxonomy, themes, and theory. Health Serv Res 2007 Aug;42(4):1758-1772 [FREE Full text] [doi: 10.1111/j.1475-6773.2006.00684.x] [Medline: $\underline{17286625]}$

82. Chenail RJ. How to conduct clinical qualitative research on the patient's experience. Qual Rep 2011;16(4):1172-1189.

83. Sandelowski M. Whatever happened to qualitative description? Res Nurs Health 2000 Aug;23(4):334-340. [Medline: $\underline{10940958]}$

84. Munro BH. Statistical Methods for Health Care Research. 5th ed. Philadelphia: Lippincott Williams \& Wilkins; 2005.

85. Holloway I, Galvin K. Qualitative Research in Nursing and Healthcare. 4th ed. NJ: Wiley-Blackwell; 2016. 


\author{
Abbreviations \\ CONSORT: Consolidated Standards Of Reporting Trial \\ CEAC: cost-effectiveness acceptability curves \\ EPDS: Edinburg Postnatal Depression Scale \\ FSSMS: Functional Social Support Measuring Subscale \\ HADS-A: Hospital Anxiety and Depression Scale \\ ICER: incremental cost-effectiveness ratios \\ IUCD: intrauterine contraceptive device \\ MPSE: Maternal Parental Self-efficacy \\ NHG DSRB: National Health Group Domain Specific Review Board \\ ODS: Ordinal Descriptive Scale \\ PMPSE: Perceived Maternal Parental Self-efficacy \\ PND: postnatal depression \\ QHSU: Questionnaire on Healthcare Services Utilization \\ SSSMS: Structural Social Support Measuring Subscale \\ SPSS: Statistical Package for the Social Sciences
}

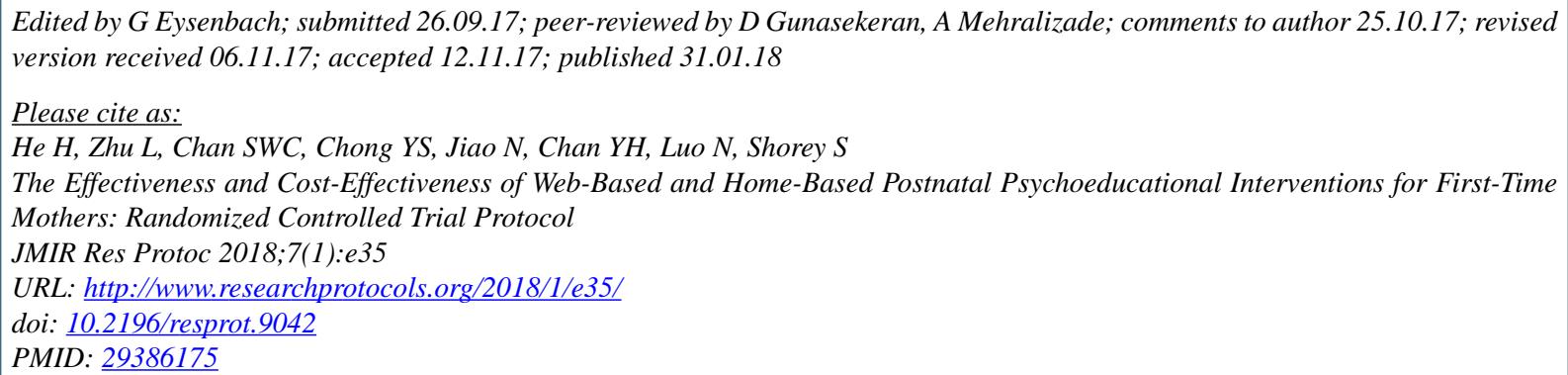

(CHonggu He, Lixia Zhu, Sally Wai Chi Chan, Yap-Seng Chong, Nana Jiao, Yiong Huak Chan, Nan Luo, Shefaly Shorey. Originally published in JMIR Research Protocols (http://www.researchprotocols.org), 31.01.2018. This is an open-access article distributed under the terms of the Creative Commons Attribution License (https://creativecommons.org/licenses/by/4.0/), which permits unrestricted use, distribution, and reproduction in any medium, provided the original work, first published in JMIR Research Protocols, is properly cited. The complete bibliographic information, a link to the original publication on http://www.researchprotocols.org, as well as this copyright and license information must be included. 